ARTÍCULO ORIGINAL

\title{
CLIMA ORGANIZACIONAL EN EL TALENTO HUMANO DE LAS INSTITUCIONES HOSPITALARIAS, MANABÍ - ECUADOR
}

\section{ORGANIZATIONAL CLIMATE IN THE HUMAN TALENT OF THE HOSPITAL INSTITUTIONS, MANABÍ - ECUADOR}

\author{
Lady Zambrano Montesdeoca \\ Universidad Nacional Mayor de San Marcos \\ *Articulo basado en la tesis doctoral del autor \\ Doctor en Ciencias Contables y Empresariales - obtenido en la Universidad Nacional Mayor de San Marcos - Lima. Magister en Ad- \\ ministración Pública - obtenido en la Universidad Tecnológica América (UNITA). Ingeniera en Administración de Empresas - obtenido \\ en la Pontificia Universidad Católica del Ecuador (PUCE). \\ Correo del autor: ay_abao@hotmail.com \\ [Recibido: 07/08/2017 Aceptado: 01/03/2018]
}

\section{RESUMEN}

El objetivo de la presente investigación es determinar la Influencia del Clima Organizacional en el Talento Humano de las Instituciones Hospitalarias de la Provincia de Manabí - Ecuador en el año 2015. Para el estudio de este ámbito de la gestión, se desarrolla un análisis de ambas variables aplicando diferentes técnicas e instrumentos adaptados a las particularidades del caso de estudio. Identificando qué componentes lo afectan y cómo se pueden perfeccionar. A partir de ello, mejorar el Clima Organizacional para obtener un mejor desempeño del Talento Humano. El diseño de la investigación es no experimental/descriptivo, de tipo correlacional, cuantitativo y transversal. Dando como resultado que el Clima Organizacional influye en el Talento Humano de las Instituciones Hospitalarias, entendiéndose que el entorno de trabajo influye en el desempeño y satisfacción del mismo. Las Normas y Reglas facilitan la División de Cargos y Salarios, lo que permite habilidad en el desempeño del trabajo. La toma de decisiones de los trabajadores está íntimamente relacionada con la responsabilidad. Los Estándares de Trabajo influyen en el servicio que brindan los empleados a los usuarios y; los trabajadores se encuentran recompensados por la labor que realizan, recibiendo un trato justo y respetuoso.

Palabras clave : Clima organizacional, Talento humano, Instituciones hospitalarias.

\begin{abstract}
The investigation determined the influence of the Organizational Climate on the Human Talent of the Hospital Institutions of the Province of Manabí - Ecuador in 2015. For the study of this area of management, an analysis of both variables is developed applying different techniques and instruments adapted to the particularities of the case study. Identifying which components affect it and how they can be perfected. From this, improving the Organizational Climate to obtain a better performance of Human Talent. The design of the research is non-experimental / descriptive, correlational, quantitative and transversal. As a result, the Organizational Climate influences the Human Talent of the Hospital Institutions, understanding that the work environment influences its performance and satisfaction. The Rules and Regulations facilitate the Division of positions and Salaries, which allows skill in the performance of work. The decision making of workers is closely related to responsibility. The Work Standards influence the service provided by employees to users and workers are rewarded for the work they do, receiving fair and respectful treatment.
\end{abstract}

Keywords :Organizational climate ,Human talent, Hospital institutions.

\section{INTRODUCCIÓN}

La investigación reconoce la influencia del Clima Organizacional en el Talento Humano de las Institucio- nes Hospitalarias de la Provincia de Manabí, para tal efecto, se formula la siguiente interrogante: ¿Cuál es la influencia del Clima Organizacional en el Talento Humano de las Institu- ciones Hospitalarias de la Provincia de Manabí - Ecuador en el año 2015? Es así que la hipótesis que responde a la pregunta es: "El Clima Organizacional existente influye en el Talento 
Humano de las Instituciones Hospitalarias de la Provincia de Manabí Ecuador en el año 2015”.

En el Ecuador se ha dado énfasis en el Talento Humano dentro de las instituciones, pues existen áreas departamentales en cada una de ellas que se encargan del manejo y organización del personal que labora en la entidad. Más aún en instituciones del área de Salud en donde se brindan servicios a la ciudadanía que deben ser de excelencia para su bienestar. Es por ello que la realización de la investigación del Clima Organizacional y del Talento Humano es importante ya que se estudian las percepciones que las personas tienen frente a un ambiente laboral.

Para la investigación se hizo uso de estudios realizados por diversos autores que fueron de gran aporte para el desarrollo de la misma, impartiendo diversos casos con sus fortalezas y debilidades que ayudaron a su realización. Resaltando que para poder alcanzar un buen rendimiento laboral, se deben tomar en cuenta muchos factores dentro de una institución, de manera especial considerar que el Talento Humano es el eje principal, el cual permite que una organización sea productiva, competitiva, que brinde productos o servicios de calidad, y logre sus objetivos institucionales; todo esto se consigue si el personal se siente en un ambiente de confort $\mathrm{y}$ adecuado al desempeño de sus funciones.

En la actualidad hablar de Clima Organizacional es una necesidad de la mayoría de los investigadores y a su vez de los profesionales que tienen a su cargo el manejo de las personas y los recursos de una entidad, pues vivimos en un mundo que crece a pasos agigantados, en donde debemos estar atentos a los grandes cambios que se presentan día a día en la práctica profesional de los cargos que se desempeñan.

El Clima Organizacional, según Castillo (2014, pág. 6), es entendido como el ambiente propio de cada organización como al conjunto de percepciones compartidas por los trabajadores en relación a dicho entorno, así mismo este ambiente ejerce influencia directa en la conducta y el comportamiento de los miembros; así como también, el Talento Humano, de acuerdo a Martha Alles (2007) son el "conjunto de las características de personalidad, devenidas comportamientos, que generan un desempeño exitoso en un puesto de trabajo. Cada puesto de trabajo puede tener diferentes características en empresas y/o mercados diferentes". Dicho de otra manera, es desempeñar sus funciones en un determinado cargo laboral para alcanzar una realización superior.

El presente estudio se justifica puesto que el Clima Organizacional abarca diversos factores de percepción y a su vez de la personalidad del ser humano; pues las personas vivimos en diferentes ambientes familiares, económicos y sentimentales, por los que actuamos según afecte cada uno de ellos en nuestras vidas. Este estudio resulta muy importante porque permite a que los colaboradores de las diversas Casas de Salud puedan expresar su forma de pensar en cuanto al entorno laboral que vive su organización y la manera en que este les beneficia o les afecta a su desempeño. A su vez la investigación servirá como una fuente a futuras investigaciones aportando conocimientos, materiales de apoyo y lectura a futuros investigadores interesados en el tema.

En el área de Salud Pública se busca brindar un servicio que satisfaga las necesidades de los usuarios internos y externos; para lo cual se está obligado a poseer conocimientos convertidores que identifiquen la influencia positiva o negativa del desempeño laboral.

La investigación estuvo dirigida a los colaboradores de las Instituciones Hospitalarias de la Provincia de Manabí - Ecuador, perteneciente a la zona 4 del Ministerio de Salud Pública del Ecuador conformado por 12 Hospitales, sin embargo se consideraron 7 , debido a que los 5 restantes son Hospitales que no tienen autonomía y dependen de una Dirección Distrital. La cifra actualizada son 2658 personas que laboran en estas Casas de Salud.

El bienestar de las personas y de manera especial la salud de ellos, es un tema primordial para una investigación, pues estamos refiriéndonos a la vida y buen desempeño del ser humano en su diario vivir. La relación entre el Clima Organizacional y el Talento Humano en Instituciones de Salud, no solo se refiere a la salud de los usuarios, sino también al bienestar de los colaboradores. Pues una vez que los colaboradores se sienten aptos para rendir un buen desempeño, los usuarios van a ser el fruto de ese buen rendimiento y entrega de calidad en la atención.

\section{Para García (2009),"z}

El Clima Organizacional entendiéndose como la percepción que tienen los trabajadores frente al entorno de trabajo en el que se desempeñan es considerado como un desafío para el estudio de los investigadores; ya que enmarca no solo el ambiente físico sino también el comportamiento de las personas frente a este.

A continuación se presenta conceptos sobre el Clima Organizacional para conocer la conceptualización de algunos autores y realizar un análisis crítico basado en sus percepciones. 
Méndez (2006) indica que "el Clima Organizacional repercute en las motivaciones que tienen los miembros de una organización, su origen está en la sociología en donde el concepto de organización dentro de la teoría de las relaciones humanas, enfatiza la importancia del hombre en su función del trabajo por su participación en un sistema social."

Chivenato (2009) dirá que "el Clima Organizacional se refiere al ambiente existente entre los miembros de la organización. Está ligado al grado de motivación de los empleados e indica de manera específica las propiedades del ambiente organizacional. Por consiguiente, es favorable proporcionar la satisfacción de las necesidades personales y la elevación moral de los miembros, y desfavorable cuando no se logra satisfacer esas necesidades."

Segredo (2010) nos dice "la comprensión del fenómeno organizacional es una necesidad de todos los profesionales que tienen alguna responsabilidad en el manejo de personas y recursos en una sociedad moderna donde el avance acelerado de la ciencia y la técnica la obliga a competir dentro de un mercado cuya dinámica está pautada fundamentalmente por el desarrollo".

Chiang (2010) indica que " Las primeras aportaciones resaltaron las propiedades o características dominando en primera instancia los factores organizacionales o situacionales. Posteriormente aparecieron un segundo grupo de definiciones que dieron mayor relevancia a las representaciones cognitivas y representaciones en los que los factores individuales son determinantes. Una tercera aproximación conceptual ha considerado el clima con un conjunto de percepciones fundamentales o globales en que se considera la interacción entre la persona y la situación.”.

Se recurrió a investigaciones ya realizadas por diversos autores en los cuales se detallan los más importantes por cada variable estudiada.

En (Flores, 2013), en su tesis titulada "Aplicación de los estímulos organizacionales para el mejoramiento del Clima Organizacional el caso del laboratorio farmacéutico corporación Infarmasa S.A", presentada por Juliana Flores Jaime. Dicha investigación fue de gran aporte, pues se pudo observar que su realidad era similar a las instituciones objeto de estudio, concluyendo que en el ambiente físico hay que realizar ciertos cambios que no permiten desempeñar a cabalidad las funciones, pues cada área de trabajo tiene sus necesidades en diferentes entornos; en cuanto a la estructura orgánica en la mayoría de los casos no se conoce, por lo que hay que recomendar que existan mayores reuniones internas y se imparta a los colaboradores las normativas, reglamentos y estructuras de la organización; darle mayor énfasis a la responsabilidad y compromiso de las personas, lo que se busca es que la mayoría preste sus servicios con la certeza de que es parte importante en la institución y que su presencia es primordial para su beneficio personal y para el logro de los objetivos institucionales. A su vez impartió una amplia conceptualización en cuanto al Clima Organizacional, estímulos organizacionales, estrategias de motivación, entre otras; que permitieron un análisis más profundo del área que se estaba estudiando.

En (Ortega, 2015), en su tesis titulada "Las Competencias Laborales y el Clima Organizacional del Personal Administrativo en las Universidades Tecnológicas Privadas de Lima”, pre- sentada por Carlos Ortega Muñoz. Se dio a conocer las diversas teorías que tienen los autores referentes al Clima Organizacional, que permitieron identificarlos según las necesidades del caso de estudio. Tomando como ejemplo para la organización e identificar mediante la observación ciertas competencias en las áreas de trabajo, que sin embargo deberían ser las mismas que estén plasmadas en un Manual de Funciones o reglamentado dentro de la institución.

Para Robbins y Coulter (2010) ,"el comportamiento organizacional se centra en tres áreas importantes; el individual, del grupo y el organizacional". Desde el punto de vista individual incorpora su personalidad, el aprendizaje y la motivación; grupalmente se relaciona con las normas, funciones, liderazgo y desde el punto de vista organizacional se refiere a la estructura, políticas y prácticas de recursos humanos". (pág. 283)

Chiavenato dirá (2011) que "las personas constituyen el capital humano de la organización. Este capital vale más o menos en la medida que contenga talentos y competencias capaces de agregar valor a la organización, además de hacerla ágil y competitiva” (pág. 58). El ser humano es el ente principal en una sociedad, quien brinda mediante su conocimiento y experiencia diversas formas de actuar frente a situaciones cotidianas.

Para García (2009) "el Clima Organizacional nace de la idea de que el hombre vive en ambientes complejos y dinámicos, puesto que las organizaciones están compuestas de personas, grupos y colectividades que generan comportamientos diversos y que afectan dicho ambiente. 
Méndez (2006) manifiesta que "el origen del Clima Organizacional se encuentra en la sociología; en donde el concepto de organización dentro de la teoría de las Relaciones Humanas enfatiza la importancia del hombre en su función del trabajo y por su participación en un sistema social”.

Según Gross (2012) "Litwin y Stinger son los primeros en plantear que el Clima Organizacional contaba con nueve componentes: Estructura, Responsabilidad o Autonomía en la toma de decisiones, Recompensa recibida, el Desafío de las metas, las Relaciones y la Cooperación entre sus miembros, los Estándares de productividad, el Manejo del conflicto y la Identificación con la Organización”. Así como el estudio de diversos autores más, de los cuales se consideraron para el estudio de la investigación los siguientes: Estructura Organizacional, Responsabilidad Laboral, Estándares de Trabajo y Recompensa por la labor.

El Talento Humano según Martha Alles (2007) en su libro titulado Desarrollo del Talento Humano Basado en Competencias, hace referencia al Talento Humano como un sinónimo de competencias, en donde define que son "las características de personalidad, devenidas comportamientos, que generan un desempeño exitoso en un puesto de trabajo. Cada puesto de trabajo puede tener diferentes características en empresas y/o mercados diferentes". Es decir, que cada institución cuenta con diferentes tipos de personas, que rinden de diferentes maneras, y desarrollan sus actividades bajo sus conocimientos y experiencias, sin embargo al momento de hablar del ser humano es considerado como una persona común y corriente, que cuenta con características personales y conocimientos profesionales, lo que permite ponerlas en práctica al momento de desarrollar sus actividades.

A continuación, se verá una serie de conceptos acerca del talento humano. Según Siliceo (1977) “es una actividad planeada, basada en las necesidades reales de una empresa y orientada hacia un cambio en los conocimientos, habilidades y actitudes del colaborador".

Para Werther \& Davis (2000) es un " Conjunto de actividades encaminadas a desarrollar habilidades y modificar actitudes del personal de todos los niveles para que desempeñen mejor su trabajo."

Según Balza(2010) "Conjunto de saberes y haceres de los individuos y grupos de trabajo en las organizaciones, pero también a sus actitudes, habilidades, convicciones, aptitudes, valores, motivaciones y expectativas respecto al sistema, organización, trabajo y sociedad.

Jericó (2011) “Como aquella gente cuyas capacidades están comprometidas a hacer cosas que mejoren los resultados en la organización.”.

Cuesta (2010) en su obra Tecnología de la Gestión de Recursos Humanos, hace referencia que para el estudio del Talento Humano se consideraron cuatro dimensiones: : división de Cargos y Salarios, autonomía en las decisiones, rendimiento laboral y percepción de equidad, tal como lo indica una vez que se dio lectura a su libro.

Martínez (2013) en su tesis titulada "Gestión del Talento Humano por competencias para una empresa de las Artes Gráficas". hace referencia a la variable del Talento Humano, la cual sirvió de base para analizar las diversas competencias que existen en las áreas de trabajo, y considerar aquellas que se asemejen al caso de estudio, pues en el área administrativa muchas veces las competencias pueden ser las mismas diferenciando la actividad de cada empresa. La autora definió las competencias, luego de un análisis en el Manual Organizacional, la misión, visión y valores de la empresa para a partir de ello diseñar diccionarios que tal como lo indica es la parte medular de cualquier Sistema de Gestión del Talento Humano por competencias.

A su vez Anaya y Paredes (2015) en su tesis titulada "Propuesta de mejora de Clima Organizacional a partir de la Gestión del Talento Humano" el cual fue presentado por los Ingenieros Lizeth del Rocío Anaya Gamarra y Juan Manuel Paredes Rivera. Dicha investigación sirvió para conocer la realidad de dicha entidad y tomar como base para realizar un plan de mejora en las Instituciones Hospitalarias que es la recomendación a la que se llegó después del estudio realizado en las Casas de Salud.

El brindar buena atención en las casa de salud, parte del ser humano, pues sin este no se puede llevar a cabo ninguna acción institucional. Se considera a las Instituciones Hospitalarias como entidades que brindan un servicio de calidad y calidez en bien de una comunidad, por lo que su funcionamiento interno debe estar en buenas condiciones para que la prestación del servicio sea el adecuado.

De las Instituciones Hospitalarias como variable interviniente, se encontraron investigaciones como la de Gamarra (2013) en su tesis titulada "Diagnóstico del Clima Organizacional y el Desempeño Laboral del Talento Humano del Hospital de Santa Elena Dr. Liborio Panchana, año 2013", presentada por Erika Gamarra. 
Al ser una Institución Hospitalaria en el mismo territorio Ecuatoriano presenta las mismas exigencias que el gobierno de planta exige a las entidades del estado. Es por eso que bajo su estudio se pudo notar las falencias que tiene para poder corregirlas y las fortalezas para poderlas adaptar a las organizaciones estudiadas.

Tomando en consideración que el Desempeño del Talento Humano es bueno o malo, dependiendo de muchos factores, siendo el principal el ambiente de trabajo en el que se desempeña.

Como también la de Borroto (2005) en su tesis titulada "Contribución al mejoramiento de la Gestión del Mantenimiento en Hospitales en Cuba”. Presentada por Yodaira Borroto Pentón. Este estudio permite comparar las formas de llevar a cabo los trabajos en la entidad estudiada por la autora y las Instituciones Hospitalarias de la Provincia de Manabí consideradas para la investigación, mencionando que el mejoramiento de los Hospitales en todas las áreas de trabajo es de beneficio organizacional y de toda una comunidad que recibe la atención de salud.

\section{MATERIAL Y MÉTODOS}

\section{Método de Investigación}

La investigación fue de diseño no experimental/descriptivo, donde la observación fue el medio primordial para llevar a cabo la investigación; el estudio fue de tipo correlacional, cuantitativo y transversal. De tipo correlacional, ya que estaba enfocada a examinar la relación entre variables o resultados de variables. Cuantitativo porque se estudió el comportamiento y los hábitos del ser humano para poder determinar su conducta fren- te al ambiente de trabajo, basado en números para dar respuestas exactas mediante la escala de Likert. Los estudios transversales se utilizaron ya que el objetivo era analizar los datos obtenidos de un grupo de personas.

\section{Instrumentos de investigación}

La encuesta fue el instrumento empleado para la investigación, ya que fue aplicada a un número determinado de personas, haciendo uso de un cuestionario que se aplicó a los funcionarios de las Instituciones Hospitalarias de la Provincia de Manabí.

\section{Población de estudio}

La población la constituye la totalidad del Talento Humano de las Instituciones Hospitalarias de la Provincia de Manabí, Ecuador, la cual pertenece a la zona 4 del Ministerio de Salud Pública del Ecuador que son 12 Hospitales, de los cuales se consideraron 7 , debido a que los 5 restantes son Hospitales que no tienen autonomía y dependen de una Dirección Distrital. Se pudiera ofrecer la cifra actualizada de 2658 personas en estas instituciones.

\section{Muestra}

El tamaño de la muestra se calculó mediante la fórmula estadística:

$$
n=\frac{N k^{2} P Q}{e^{2}(N-1)+k^{2} P Q}
$$

Dando como resultado un total de 189 personas a las cuales se les realizó la encuesta.

El procedimiento o método de muestreo fue probabilístico de tipo aleatorio estratificado, que incluye una muestra representativa del personal administrativo, médicos, enfermeras, paramédicos y personal de apoyo que laboran en las Instituciones Hospitalarias de la Provincia de Manabí.

\section{Coeficiente de Correlación Rho de Spe- arman}

Se empleó a la prueba de Rho de Spearman puesto que los datos son no paramétricos, siendo el coeficiente que permite medir la correlación o asociación entre dos variables cuando las mediciones se realizan en una escala ordinal, o cuando no existe distribución normal.

La fórmula del estadístico es de la siguiente manera, en su forma estándar:

$$
\rho=\frac{\sum_{i=1}^{n}\left(x_{i}-\bar{x}\right)\left(y_{i}-\bar{y}\right)}{\sqrt{\sum_{i=1}^{n}\left(x_{i}-\bar{x}\right)^{2} \sum_{i=1}^{n}\left(y_{i}-\bar{y}\right)^{2}}}
$$

Dónde:

$\rho$ : Coeficiente de correlación

$\mathrm{N}$ : Número de pares ordenados

$\mathrm{X}$ : Variable (X)

Y: Variable $(\mathrm{Y})$

Por tanto, si el valor $\mathrm{p}>0.05$, se acepta la hipótesis nula. En caso contrario, si el valor de $\mathrm{p}<=0.05$, se rechaza la nula, aceptando la hipótesis alternativa.

\section{Coeficiente de correlación Tau_b de Kendall}

Se empleó la prueba de Tau-b de Kendall que es una técnica no paramétrica para medir el grado de correlación entre variables de una muestra, este coeficiente mide el grado de asociación o concordancia entre varios conjuntos de datos. 


\section{RESULTADOS}

Para demostrar los resultados de la investigación a continuación se detallan las variables estudiadas, las que permitieron determinar la influencia que tiene el Clima Organizacional en el Talento Humano de las Instituciones Hospitalarias de la Provincia de Manabí como base principal para el estudio de la presente investigación.

Haciendo uso de la escala de Likert para poder ponderar sus respuestas:
1. Totalmente en desacuerdo.

2. En desacuerdo.

3. Indiferente.

4. De acuerdo.

5. Totalmente de acuerdo.

\section{$\mathrm{X}=$ Clima Organizacional}

$\mathrm{X} 1$ = Estructura Organizacional

$\mathrm{X} 2$ = Responsabilidad Laboral

X3 = Estándares de Trabajo

X4 = Recompensa por la Labor

\author{
$\mathrm{Y}=$ Talento Humano \\ Y1 = División de Cargos y Salarios \\ Y2 = Autonomía en las Decisiones \\ Y3 = Rendimiento Laboral \\ Y4 = Percepción de Equidad
}

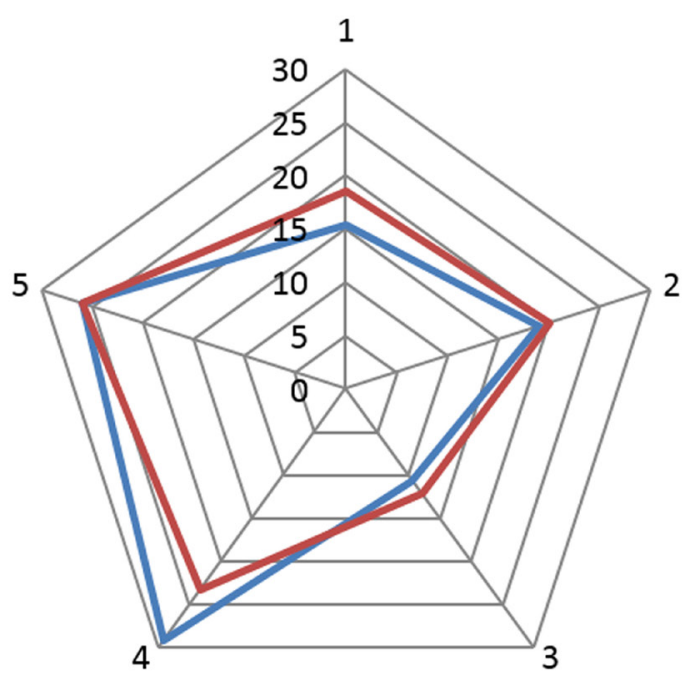

ESTRUCTURA
ORGANIZACIONAL

—DIVISIÓN DE CARGOS Y

SALARIOS

Figura 1. Relación de la Estructura Organizacional con la División de Cargos y Salarios, Fuente: Elaboración propia.

\section{Interpretación (Figura 1)}

Los resultados obtenidos según la encuesta reflejó que en cuanto a la Estructura Organizacional el $15 \%$ contestaron que están totalmente en desacuerdo, el $19 \%$ en desacuerdo, el $11 \%$ indiferente, el $29 \%$ de acuerdo y el $26 \%$ totalmente de acuerdo; a su vez en cuanto a la División de Cargos y Salarios, el 19\% contestó que están totalmente en desacuerdo, el $20 \%$ en desacuerdo, el $12 \%$ indiferente, el $23 \%$ de acuerdo y el $26 \%$ totalmente de acuerdo, lo que quiere decir, que en las Instituciones Hospitalarias estudiadas, las Normas y Reglamentos dentro de las entidades no están sien- do impartidas a sus colaboradores; el entorno laboral hay que mejorarlo tanto en infraestructura y la parte interna, de esta manera las personas sabrán diferenciar la división de cargos dentro de la organización, consiguiendo de esta manera que el personal brinde un servicio de calidad a los usuarios. (Ver Figura 2) 


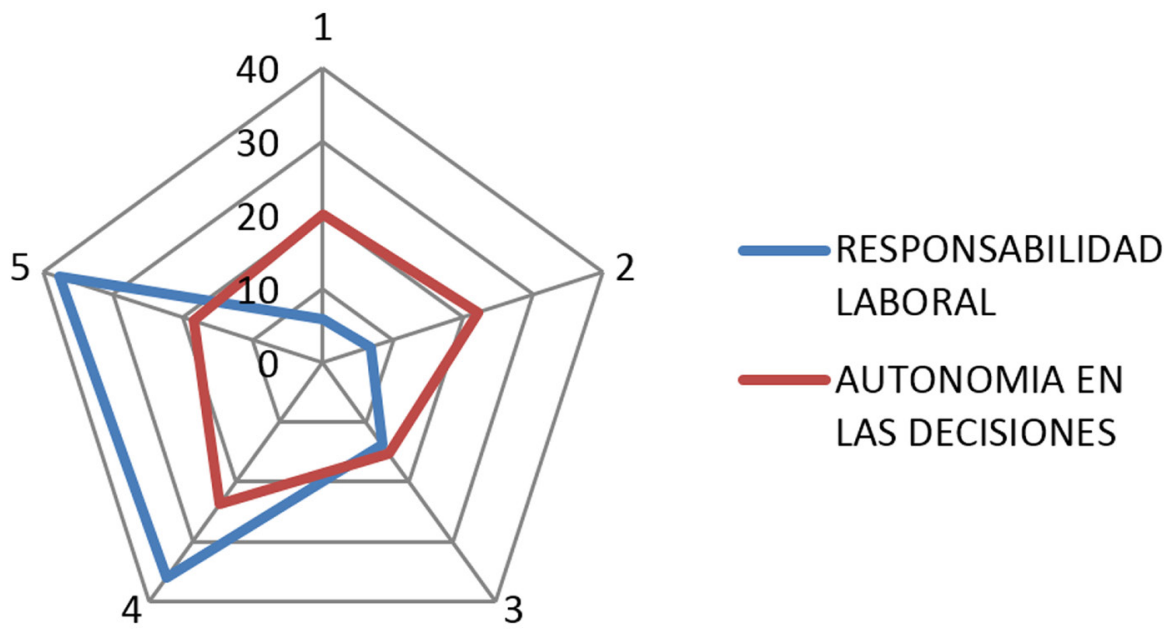

Figura 2. Relación de la Responsabilidad Laboral con la Autonomía en las decisiones. Fuente: Elaboración propia.

Interpretación (Figura 2)

En cuanto a la Responsabilidad Laboral el $6 \%$ estaban totalmente en desacuerdo, el $7 \%$ en desacuerdo, el $14 \%$ indiferente, el $36 \%$ de acuerdo y el $38 \%$ totalmente de acuerdo; a su vez en lo que se refiere a la Autonomía en las decisiones el $20 \%$ contestaron que están totalmente en desacuerdo, el $22 \%$ en desacuerdo, el $15 \%$ indiferente, el 24\% de acuerdo, y el 19\% totalmente de acuerdo, lo que quiere decir que el cumplimiento de las responsabilidades que tienen los colaboradores debe estar en función a las funciones encomendadas, de esta manera se podrían tomar decisiones acertadas frente a problemas que se presenten en el camino.(ver figura 3)
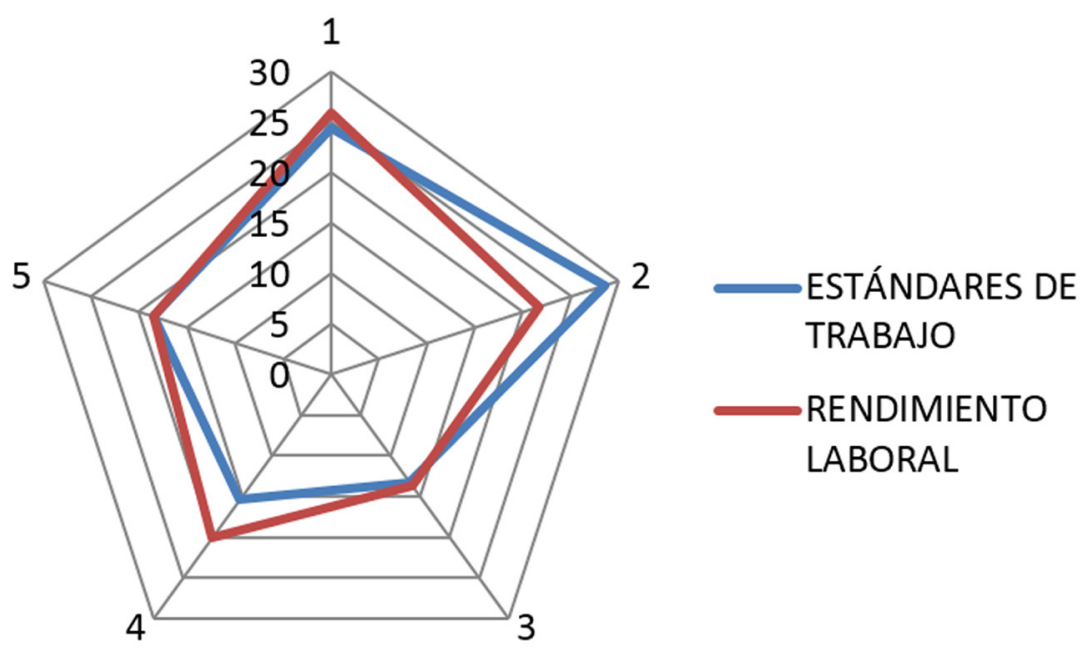

Figura 3. Relación de los Estándares de Trabajo y el Rendimiento Laboral. Fuente: Elaboración propia. 


\section{Interpretación (Figura 3)}

Los Estándares de Trabajo dieron como resultado que el $24 \%$ contesto que está totalmente en desacuerdo, el $29 \%$ en desacuerdo, el $13 \%$ indiferente, el $15 \%$ de acuerdo y el $19 \%$ total- mente en desacuerdo; frente al Rendimiento Laboral contestaron que el $26 \%$ está totalmente en desacuerdo, el $22 \%$ de acuerdo, el 14\% indiferente, el $20 \%$ de acuerdo y el $19 \%$ totalmente de acuerdo; lo que quiere decir que el personal que labora en las Institu- ciones Hospitalarias consideró que hay complejidad de sus labores debe ir en función de lo que realizan, reconociendo el trabajo que desempeñan y dándole el valor de importancia que tiene frente al rendimiento a su labor. (ver figura 4)

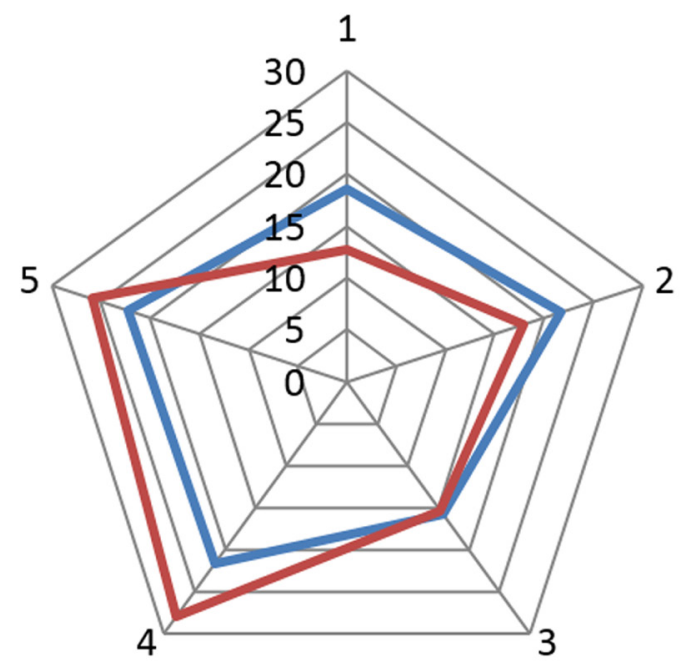

$\longrightarrow$ RECOMPENSA POR LA LABOR $\longrightarrow$ PERCEPCION DE EQUIDAD

Figura 4. Relación de la Recompensa por la Labor y la Percepción de Equidad. Fuente: Elaboración propia.

\section{Interpretación (Figura 4)}

En cuanto a la Recompensa por la Labor los resultados reflejan que el 19\% contesto que está totalmente en desacuerdo, el $22 \%$ en desacuerdo, el 16\% indiferente, el $22 \%$ de acuerdo y el $22 \%$ totalmente en desacuerdo; frente a la Percepción de Equidad contestaron que el $13 \%$ está totalmente en desacuerdo, el 18\% de acuerdo, el 15\% indiferente, el $28 \%$ de acuerdo y el $26 \%$ totalmente de acuerdo; lo que quiere decir que el ambiente laboral debe ser el acorde, rigiéndose en las normas de Seguridad y Salud Laboral presentes en el Ecuador, reconociendo la labor de sus colaboradores de manera equitativa; evaluando el desempeño de sus funciones periódicamente y así corregir las falencias que se observan.

\section{DISCUSIÓN}

El Clima Organizacional influye en el Talento Humano de las Instituciones Hospitalarias estudiadas, debido a que la carencia de un buen ambiente de trabajo, en donde se respire el compañerismo entre directivos $y$ colaboradores en general, un entorno físico adecuado y demás factores que son percibidos por el ser humano, hacen que el Talento Humano no desempeñe sus labores a cabalidad; pues lo que se busca en dichas casas de Salud y en organizaciones en general es brindar optimas atenciones a los usuarios internos y externos, manteniendo al personal satisfecho en su área de trabajo. Considerando que las normativas, leyes, reglamentos y planes de mejoramiento se deben dar a conocer a los colaboradores para que sean puestos en práctica y no exista un desconocimiento de aquello.

La Estructura Organizacional de las Instituciones Hospitalarias de la Provincia de Manabí, objeto de estudio, brindaron variedad de resultados mediante la encuesta estableciendo que las Normas y Reglamentos dentro de una organización deben ser conocidos por sus trabajadores y puestos en práctica al momento de desempeñar sus actividades, teniendo en claro la asignación de las funciones de cada departamento o área de trabajo para desempeñarse de manera organizada, evitando desperdicios de tiempo, dinero e intelecto, considerando un buen ambiente de trabajo, tanto interno como externo; de esta manera 
las personas conocerán correctamente cual es la división de los puestos y salarios para poder desempeñarse de la mejor manera y brindar un servicio de calidad a los usuarios, que es lo que buscan las personas al momento de acudir a una casa de salud.

El personal de las Instituciones Hospitalarias considera que la complejidad de sus labores debe ir acorde a lo que realiza, reconociendo el trabajo desempeñado y dándole la importancia que los colaboradores deben tener dentro de la institución; considerando que no es un gasto cuando de capacitaciones se refiere, sino más bien una inversión que la institución realiza con sus trabajadores para desarrollar mayores habilidades y ampliar el conocimiento en sus áreas de trabajo, sin dejar a un lado que la experiencia es un punto a favor que tienen las personas, pues con ella se obtiene una mayor productividad en el rendimiento laboral, y de esta forma los usuarios obtendrán atención de calidad, evitando consigo las quejas de la institución y sus miembros.

Finalmente se demostró que las condiciones de trabajo deben ser las adecuadas para desempeñar las actividades de manera segura, cumpliendo con las normativas de Seguridad y Salud Laboral como lo requieren en las Instituciones Públicas en el Ecuador, y más aún en las casas de Salud donde se debe tener las herramientas necesarias para su óptimo funcionamiento; sin dejar a un lado que el sentido de recompensa que tiene el trabajador influye en el desempeño laboral; demostrando un trato justo y equitativo de todo el personal, sin distinguir rangos estructurales de la organización; así como también la realización de evaluaciones periódicas que vayan en función de su trabajo realizado.

\section{REFERENCIAS BIBLIOGRÁFICAS}

Álvarez, G. (1992). El constructo "clima organizacional”: concepto, teorías, investigaciones y resultados relevantes. Revista Interamericana de Psicología Ocupacional, 11(1,2), 27-30.

Alles, M. (2007). Desarrollo del talento humano: basado en competencias. $3^{\mathrm{a}}$ Ed. Buenos Aires: Granica.

Anaya, L., \& Paredes, J. (2015). Propuesta de mejora de clima organizacional a partir de la gestión del talento humano, Lambayeque: Universidad del Pacífico.

Borroto, Y. (2005). Contribución al mejoramiento de la gestión del mantenimiento en hospitales en Cuba. Aplicación en hospitales de la provincia Villa Clara. (Tesis de Doctorado).: Universidad Central Marta Abreu: Pentón, Yodaira

Castillo, N. (2014). Clima, Motivación Intrínseca y Satisfacción Laboral en Trabajadores de Diferentes Niveles Jerárquicos, Lima, Pontificia Universidad Católica del Perú

Chiavenato, I. (2011). Administración de Recursos Humanos. El Capital humano de las organizaciones. 9na Edic. México D.F.: Mc Graw Hill.

Cuesta, A. (2010). Tecnología de la gestión de recursos humanos (2da edición). La Habana : Editorial Academia.
Flores, J. (2013). Aplicación de los estímulos organizacionales para el mejoramiento del clima organizacional el caso del laboratorio farmacéutico corporación Infarmasa S.A. Lima: Universidad Nacional Mayor de San Marcos.

Gamarra, E. (2013). Diagnóstico del clima organizacional y el desempeno laboral del talento humano del Hospital de Santa Elena Dr. Liborio Panchana, año 2013. (Tesis de Licenciatura). La Libertad, Ecuador: Universidad Estatal Península de Santa Elena.

Martínez, V. (2013). Gestión del talento humano por competencias para una empresa de las Artes Gráficas. México D.F: Instituto Politécnico Nacional.

Méndez, R. (2006). Clima organizacional en Colombia. El IMCOC: Un método de análisis para su intervención. Colección de lecciones de administración. Bogotá: Universidad del Bosario.

Ortega, C. (2015). Las competencias laborales y el clima organizacional del personal administrativo en las universidades tecnológicas privadas de Lima. Lima: Universidad Nacional Mayor de San Marcos.

Robbins, S., \& Coulter, M. (2010). Administración ( $10^{\mathrm{a}}$ edición $)$. México D.F: Pearson. 